\title{
Does the Nerium oleander extract PBI-05204 have potential for pancreatic cancer therapy?
}

\author{
José Manuel Calderón-Montaño • \\ Estefanía Burgos-Morón • Emilio Guillén-Mancina • \\ Miguel López-Lázaro
}

Received: 20 December 2014 / Accepted: 22 December 2014 /Published online: 21 January 2015

(C) Springer Science+Business Media New York 2015

\section{Dear Sir:}

We read with great interest the article "PBI-05204, a supercritical $\mathrm{CO} 2$ extract of Nerium oleander, inhibits growth of human pancreatic cancer via targeting the PI3K/mTOR pathway", published in Investigational New Drugs 2014 (doi:10. 1007/s10637-014-0190-6). In this article, the authors report that this cardiac glycoside-containing extract induces a remarkable antitumor effect in mice transplanted with human pancreatic cancer cells. Because patients with pancreatic cancer lack effective therapies, these data provide support for PBI-05204 as a potential therapeutic agent for pancreatic cancer that warrants further clinical investigation [1].

It has been known for some time that rodent cells are highly resistant to the cytotoxicity of cardiac glycosides. For example, we have reported that rodent cells are extremely resistant (over 1000-fold) to the cytotoxicity of these compounds when compared to human malignant and nonmalignant cells [2]. This means that it is relatively easy to observe a marked in vivo anticancer activity if one transplants human cancer cells into mice and then treats the animals with a cardiac glycoside (or with a cardiac glycoside-containing extract). This antitumor activity, however, is probably caused by the ability of these natural products to selectively kill human cells versus rodent cells rather than by their ability to selectively kill human cancer cells versus human healthy cells [2].

We have observed recently that rodent cells are over 100 times more resistant than human cells to the cytotoxicity of an extract from the leaves of Nerium oleander [3]. The selectivity

J. M. Calderón-Montaño • E. Burgos-Morón · E. Guillén-Mancina · M. López-Lázaro $(\bowtie)$

University of Seville, Seville, Spain

e-mail: mlopezlazaro@us.es of our cardiac glycoside-containing extract towards human cancer cells was much lower or nonexistent when compared to human healthy cells. Its selectivity was 10 -fold when tested in human lung cancer cells (A549) versus human lung nonmalignant cells (MRC5), but it was very low or lacking when tested in human melanoma cells (UACC62) and colorectal cancer cells (HT29) versus the nonmalignant cells [3].

The results reported by Pan et al. [1] are useful to understand the in vivo mechanism of action and pharmacokinetic profile of the Nerium oleander extract. However, additional experiments should be conducted before considering testing this extract in patients with pancreatic cancer. These experiments should reveal whether or not the extract improves the ability of the current therapies to kill human pancreatic cancer cell lines without significantly affecting human nonmalignant cell lines from a variety of appropriate tissues. This information would help us answer the question raised in the title of this manuscript.

\section{References}

1. Pan Y, Rhea P, Tan L, Cartwright C, Lee HJ, Ravoori MK, Addington C, Gagea M, Kundra V, Kim SJ, Newman RA, Yang P (2014) PBI05204, a supercritical CO2 extract of Nerium oleander, inhibits growth of human pancreatic cancer via targeting the PI3K/mTOR pathway. Invest New Drugs. doi:10.1007/s10637-014-0190-6

2. Calderón-Montaño JM, Burgos-Morón E, López-Lázaro M (2014) The in vivo antitumor activity of cardiac glycosides in mice xenografted with human cancer cells is probably an experimental artifact. Oncogene 33(22):2947-2948. doi:10.1038/onc.2013.229

3. Calderón-Montaño JM, Burgos-Morón E, Orta ML, Mateos S, LópezLázaro M (2013) A hydroalcoholic extract from the leaves of Nerium oleander inhibits glycolysis and induces selective killing of lung cancer cells. Planta Med 79(12):1017-1023. doi:10.1055/s-0032-1328715 